\title{
The Influence of Gender and Age on the Outcomes of and Adherence to an Interdisciplinary Digital Mental Health Promotion Intervention in an Australasian Nonclinical Setting: A Cohort Study
}




\title{
Section 1:
}

\section{The Website}

\author{
Images of the website 2018 \\ (e-learning management system) \\ https://eliawellness.com/
}




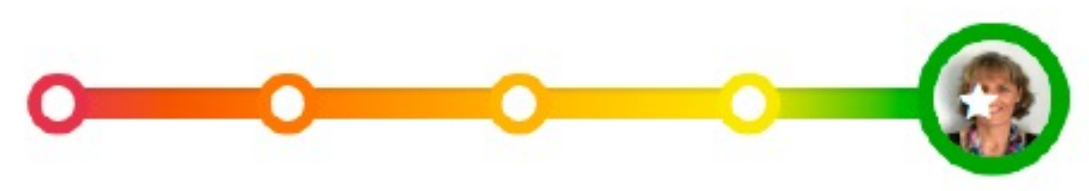

HARM

HEAL

To Do List

ELIR. View your wellness snapshot

LIFT: Log Your Your Daily Challenge

Weekly Challenge Completed

To Do List: An online "To Do" list (available on each personal dashboard) provided easily accessible links to complete the various components. Note: the "wellness snapshot" refers to the prequestionnaire administered at baseline and again on completion (postquestionnaire) of the program. 
$\oplus$ 1. Your Limbo Is Listening

(2. Motion Creates Emotion

3. Blue \& Green Should Often Be Seen

B. 4. Together Feels Better

5. Feelings Follow Your Focus

(1) 6. Food Feeds Your Mood

(32) 7. Rest To Feel Your Best

8. Stress Less

(2) 9. Giving Is Living

(8) 10. What Does It Take To Flourish?

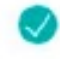

2

2

2

2

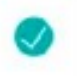

2

2

2

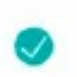

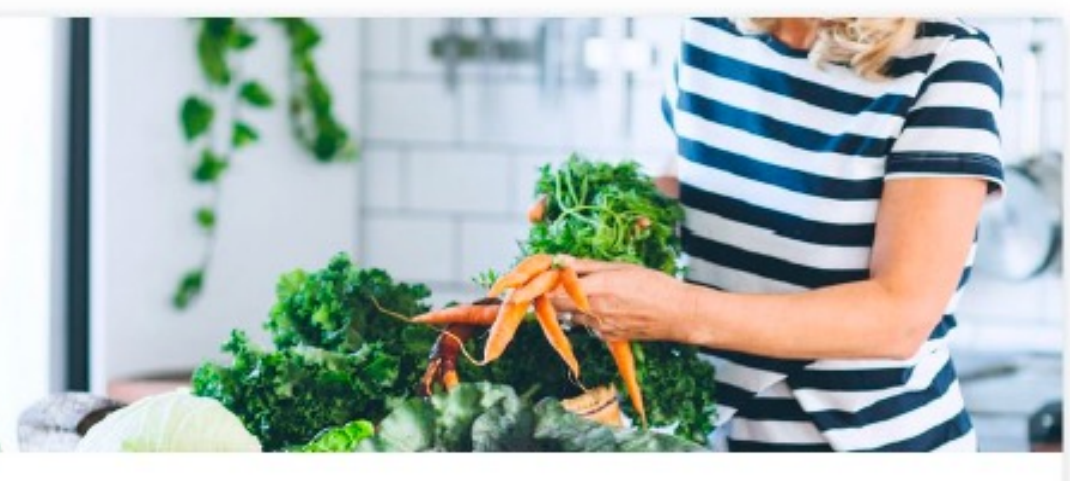

Food Feeds Your Mood

Learn how one of the most intriguing areas of medical research shows how certain types of foods that can keep the blues away.

- Introduction

- Food Feeds Your Mood

D Wrap

- Masterchef Me Extra $\nabla$

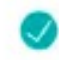

$\nabla$

2

Sample View of Lesson 6 Content: Each lesson was divided into 3-4 short video presentations. Each new video segment was unlocked, on completion of the previous video. Segments were ticked off automatically as they were viewed. 
Monday, 22 October 2018

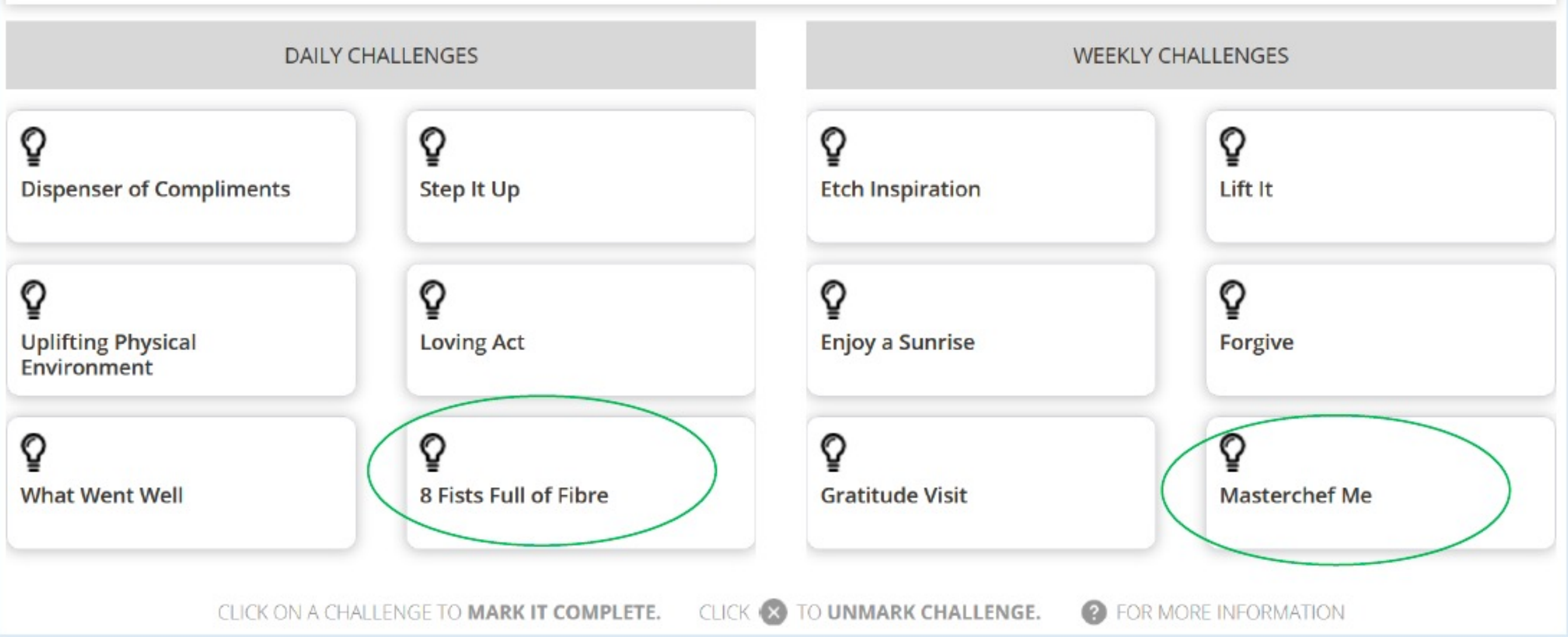

View of challenge icons for lessons 1-6: After each lesson, participants are provided with daily and weekly challenges. For example, for Lesson 6 , participants were challenged to eat 8 fists full of fibre daily, and were asked to make 1 high-fibre plant based meal for the weekly challenge - "Masterchef Me". 


\section{\} 8 Fists Full of Fibre}

Consume eight or more serves of high fibre whole foods the size of your fist. Now the best source of fibre are

fruits, vegetables, beans and grains. Wholegrain bread counts, but not white bread.

\section{(10) POINTS}

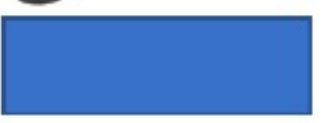

My Experience

[0] Add Photo

\section{Masterchef Me}

Prepare and share a high fibre plant based meal.

\section{POINTS}

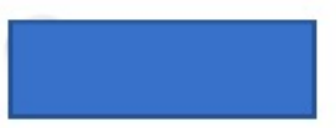

I made a Lentil and Mushroom Stew and shared with my family

941 Characters left.

[0] Update Photo

\section{MARK AS COMPLETE}

View of Lesson 6 Challenge Log Pages: When participants clicked on the challenge icon (previous image) it opened up a screen to log daily challenges (possibility of 10 points scored daily) and weekly challenges (30 points scored per week). Name and photo ID has been obscured for privacy reasons 

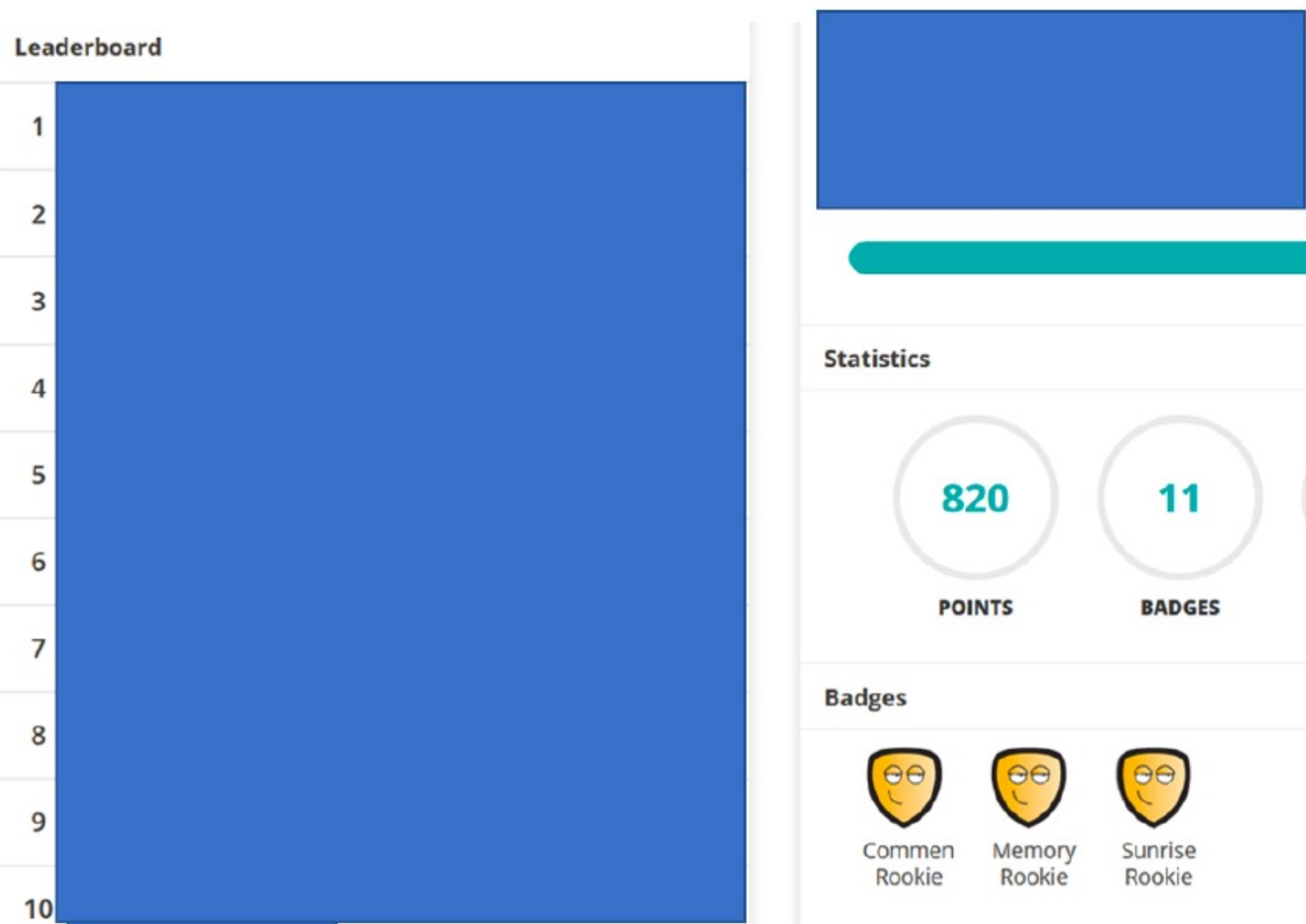

Statistics

Entire Program

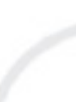

820

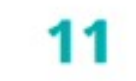

\section{8}

POINTS

BADGES

CHALLENGES

Badges

Entire Program

View of Leaderboard Names and photos have been blocked to protect privacy. Participants could compare their scores with others and earn badges for completing activities 


\section{Section 2:}

The App

The following images are from the mobile app called "mywellness" (2018) 


Abollness Assessment
Emotionally Thriving
Save your progress and continue at a later time.
Physically Energised
Socially Connected
Vocationally Enriched
Intellectually Engaged

\section{.ul Telstra $4 \mathrm{G} \quad$ 4:15 pm
Save \& Exit Socially Connected}

\section{I can talk about my problems with my friends.}

12 of 17 questions

\section{Strongly disagree}

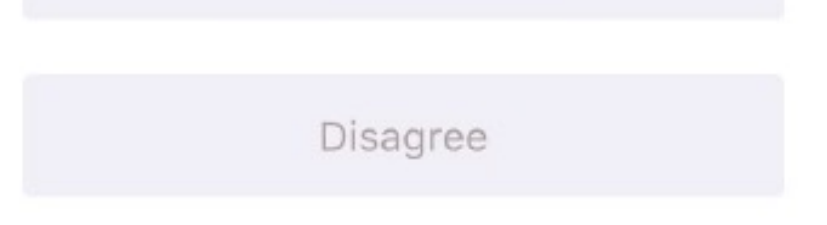

Slightly disagree

Neither agree or disagree

\section{Slightly agree}

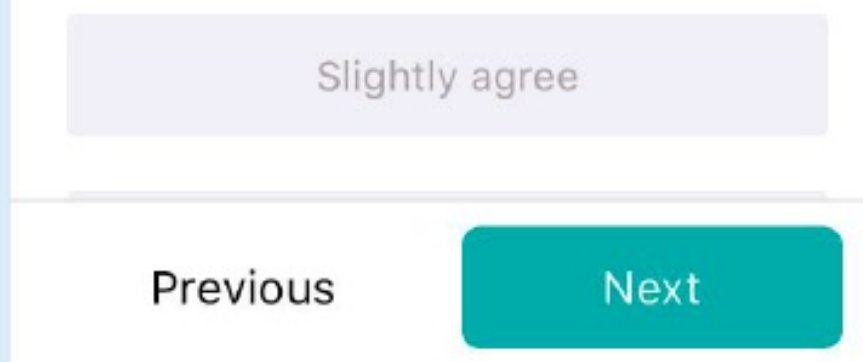

Postquestionnaire sample: No slides are available of the qualitative questions, however participants read the statement about what made it easy or hard to adhere, and were then provided with an expanding text-box to type an answer (length unlimited) 


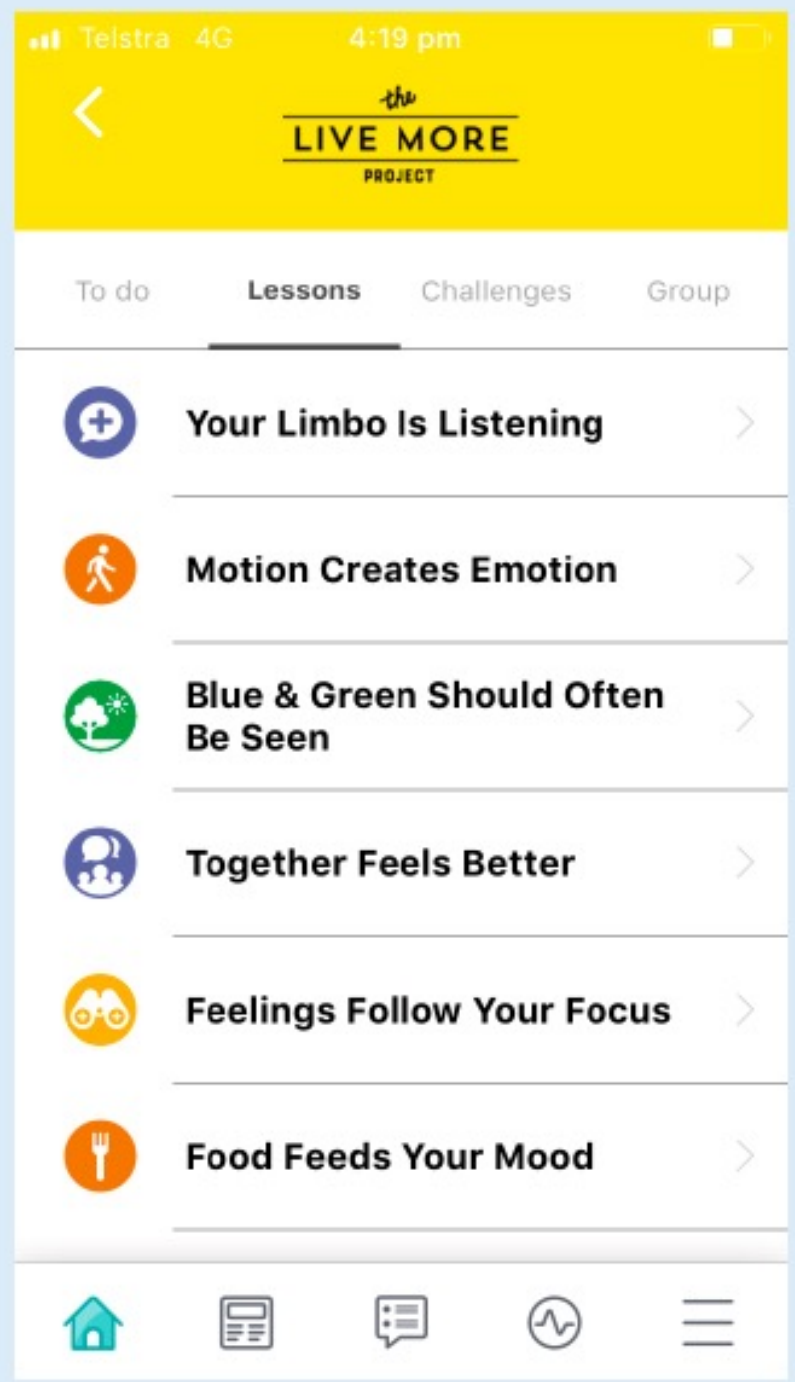

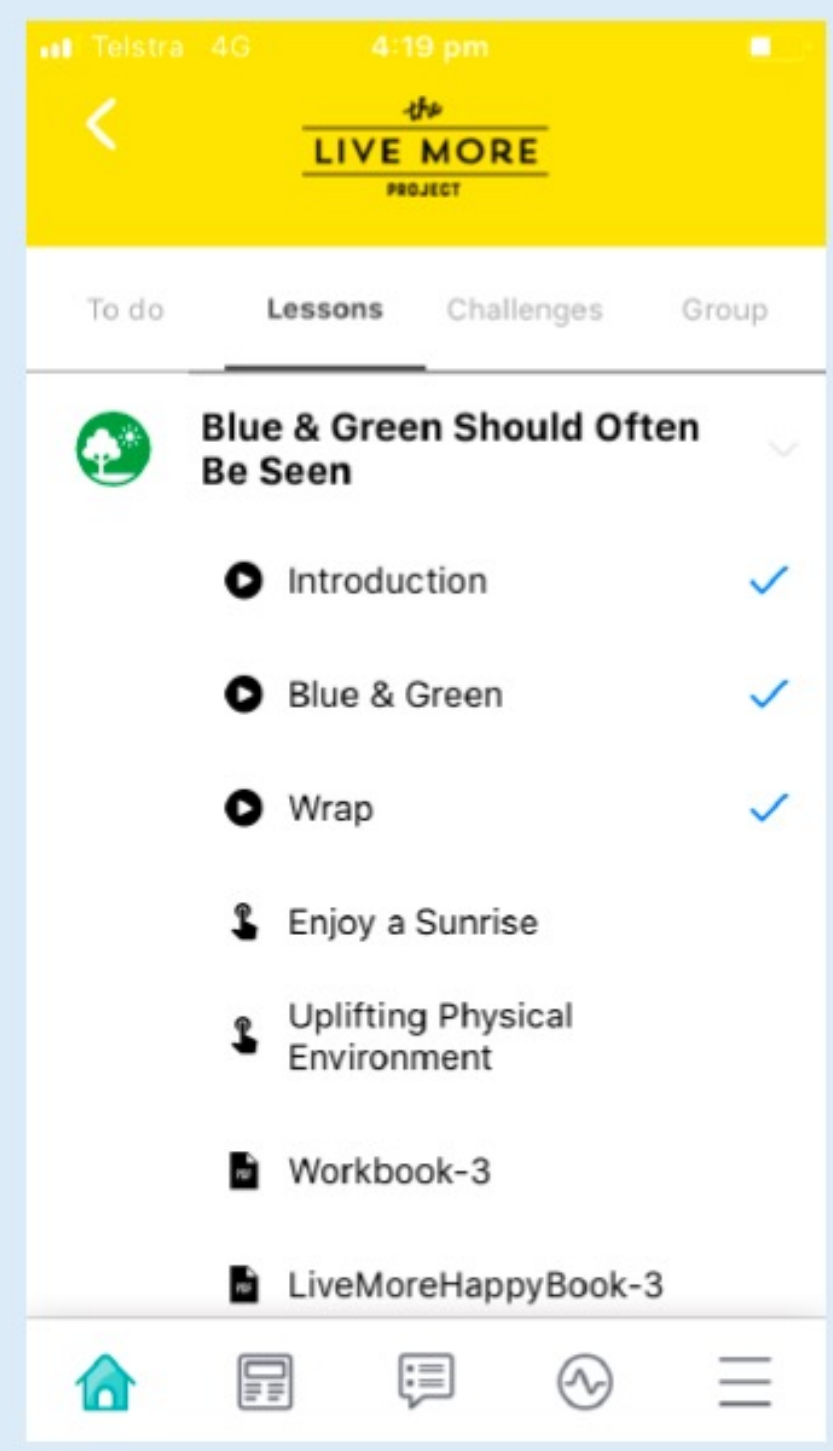

Video content on the mobile app: Participants watched the video content and could download a workbook and a chapter of the accompanying text-book book "Live More Happy" (Morton, 2018) from the Lessons page on the app (or the website). 


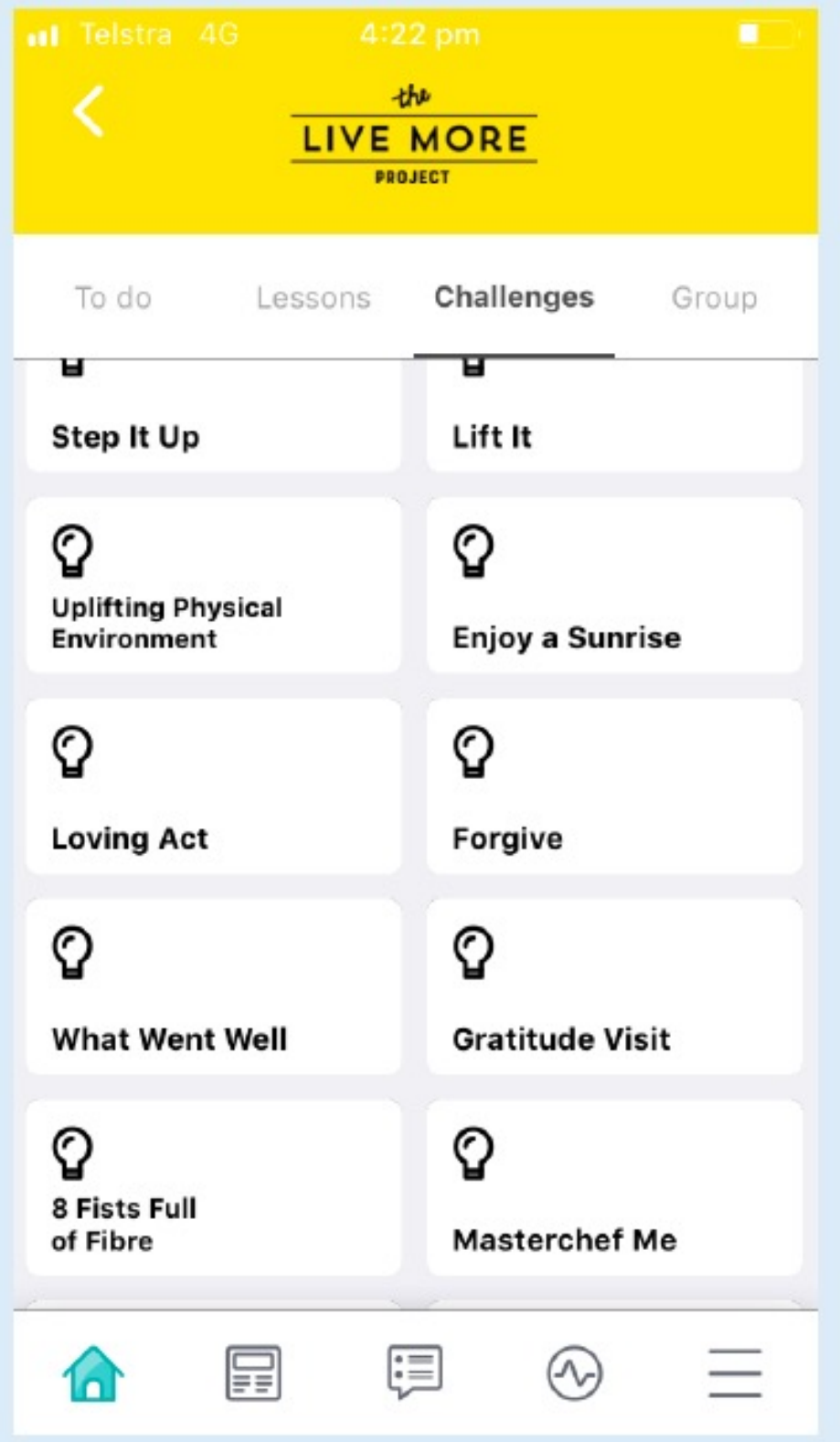

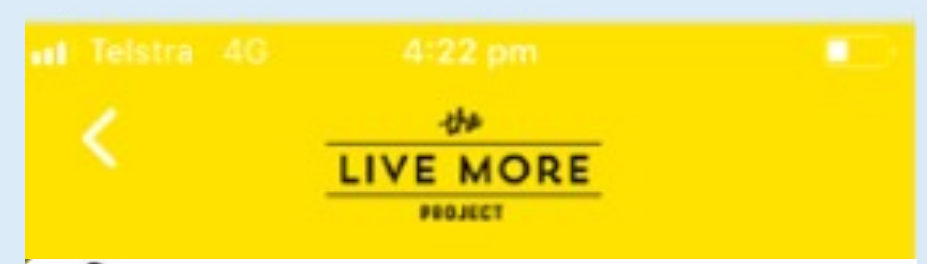

\section{Step It Up}

Complete 30 minutes of moderate intensity physical activity, remember moderate intensity is a 3 or 4 out of 10 for effort.

\section{( 10 plar points}

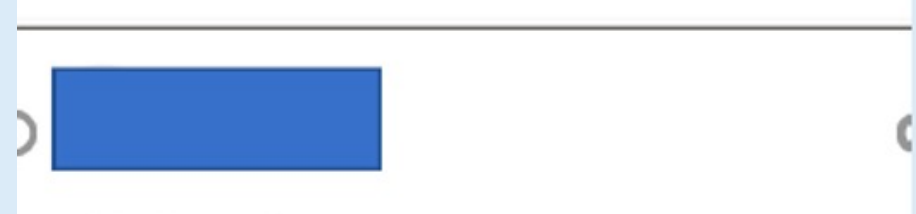

My Experience...
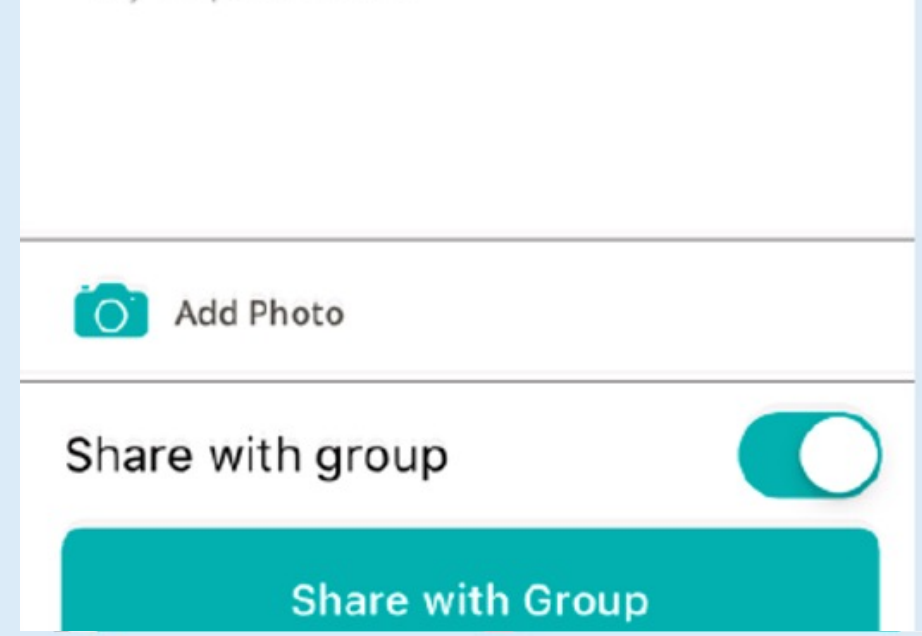

Ho LIVE MORE moster

\section{Lift It}

Perform a resistance exercise session which can include stretching, small weights, pushups, sit-ups and more.
(D) Play
30 POINTS

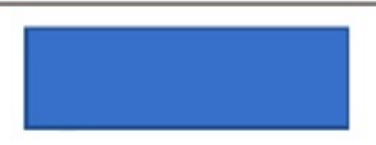

My Experience...

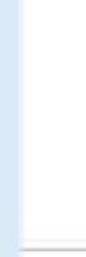

O. Add Photo

Share with group

\section{Share with Group}

Challenges on the App: Participants could click on the icon and then record challenge activity while 'on the go'. The 'blue' box is simply to de-identify personal information. 

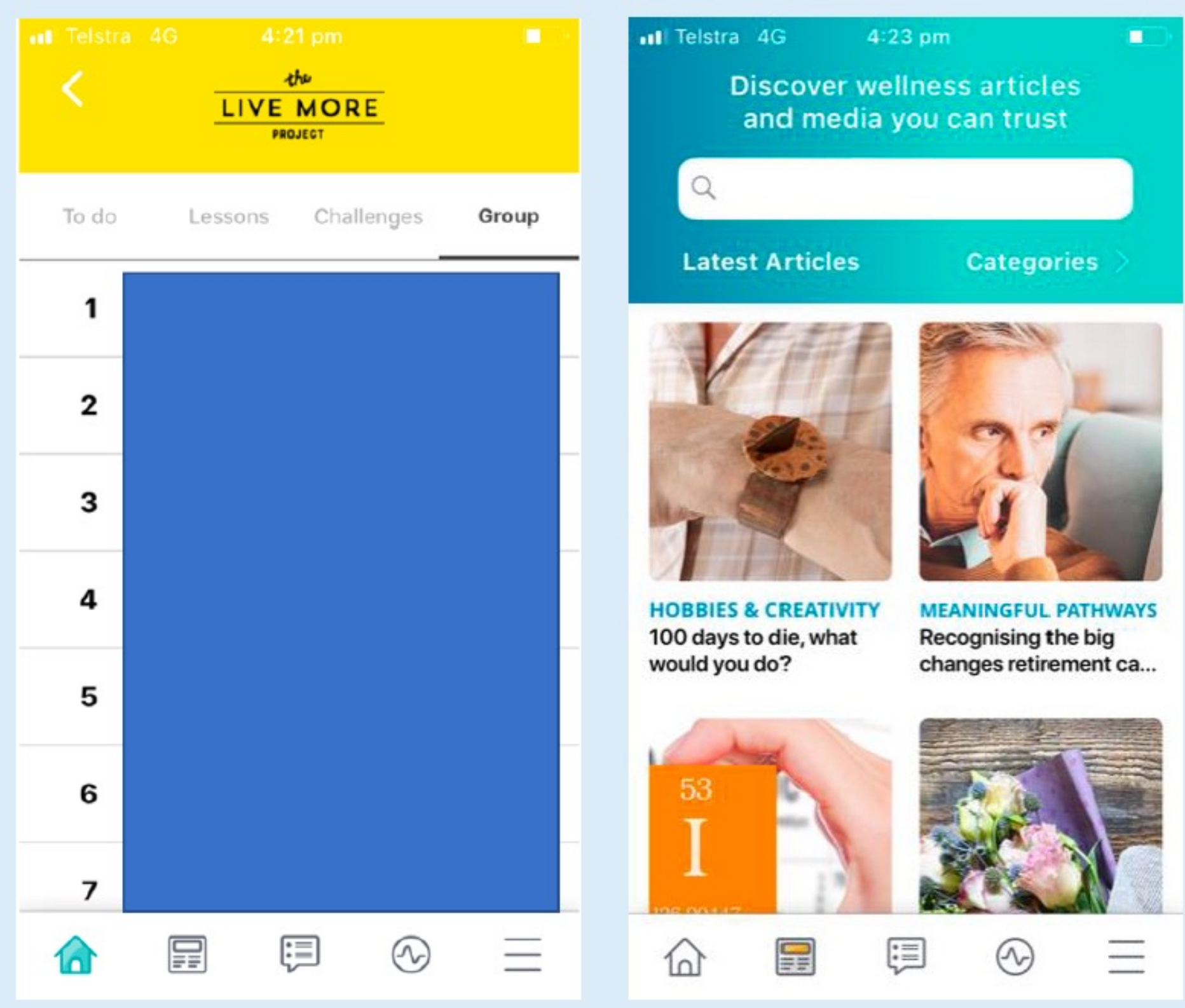

Leaderboard and extra articles: Names and photos have been removed for privacy. Participants could compare their progress with others on the leaderboard and read articles of interest related to the topics of the program. 


\section{References}

Morton D, Live more happy : scientifically proven ways to lift your mood and your life. Warburton, Vic.: Signs Publishing Company; 2018; ISBN:9781925044720. 